Europhys. Lett., 52 (6), pp. 620-626 (2000)

\title{
Magnetic-field effects on coherent backscattering of light in case of Mie spheres
}

\author{
R. Lenke ${ }^{1,2}$, R. LehNeR ${ }^{1}$ and G. MARET ${ }^{1}$ \\ 1 University of Konstanz - Box 5560, D-78457 Konstanz, Germany \\ 2 Institut Charles Sadron - 6 rue Boussingault, F-67083 Strasbourg Cedex, France
}

(received 21 June 2000; accepted in final form 17 October 2000)

PACS. 42.25.Dd - Wave propagation in random media.

PACS. 42.25. Hz - Interference.

PACS. 78.20.Ls - Magneto-optical effects.

\begin{abstract}
Magneto-optical Faraday rotation destroys the coherent backscattering enhancement of elastically multiple scattered light. In addition, Faraday rotation has been predicted, especially for Mie scattering, to cause a shift of the coherent backscattering cone off the exact backscattering direction in case of circular polarized light, or a twin peak in case of linear polarization (see Lenke R. and Maret G., Eur. Phys. J. B, 17 (2000) 171). In this letter we report the first experimental observations of these predictions. Moreover, we have observed an unexpected transversal asymmetry in the backscattered intensity, i.e. an asymmetric photon flux perpendicular to the external magnetic field and perpendicular to the direction of the incident light.
\end{abstract}

Coherent backscattering (CB) has experienced growing interest during the last years in the context of the research for a better understanding of light propagation in turbid media (for more information the reader is referred to [1-5], for example). CB originates from the constructive interference, in the exact backscattering direction, between each light path and its reversed path as both paths always have exactly the same length. CB is a very stable effect; it is not destroyed by absorption, nor by the movement of the scatterers, nor by a short coherence length of the light; in fact, $C B$ can be observed even with sunlight. Off the exact backscattering direction a phase shift of $\vec{q}_{\mathrm{b}} \cdot \vec{R}$ is introduced between each pair of light paths, where $\vec{q}_{\mathrm{b}}:=\vec{k}_{\text {out }}-\left(-\vec{k}_{\text {in }}\right)$ is the backscattering vector of light with wave vector $k=2 \pi / \lambda_{0}$ ( $\lambda_{0}$ wavelength in air); $\vec{R}$ is the distance vector between the starting and the end point of a certain multiple scattering light path. Thus, on average over all light paths, the twofold intensity enhancement of CB decreases with increasing $\vec{q}_{\mathrm{b}}$ to the intensity obtained by the non-coherent superposition of all light paths. The width of this so-called CB cone, $\left(\Delta q_{\mathrm{b}}\right)_{\mathrm{FWHM}} \approx 1 / \ell^{\star}$, is determined by the turbidity, $1 / \ell^{\star}$, of the medium. The length $\ell^{\star}$ is the transport mean free path, i.e. the characteristic length of the random walk of the light in the turbid medium. This scalar wave model does not include the vector character of light. In fact, according to the theorem of reciprocity, the amplitudes on the direct and reversed paths are only the same if the incident and detected polarization states are the same. For 
orthogonal polarization states, no or only a small $\mathrm{CB}$ cone is observed depending on the type of scattering.

Faraday Rotation (FR) is the only effect (besides relativistic effects or inelastic scattering) which destroys $\mathrm{CB}[6,7]$. FR introduces a phase shift $\alpha=\sigma V \vec{B} \cdot \vec{r}$ for circular polarized light propagating a distance $\vec{r}$ in a magnetic field $\vec{B} ; V$ is the specific Verdet constant $\left({ }^{1}\right)$ and $\sigma= \pm 1$ is the handedness of the circular polarization. Consequently, a phase difference of $\Delta \alpha=2 \sigma V \vec{B} \cdot \vec{r}$ is introduced between the light propagating parallel and anti-parallel to a path segment $\vec{r}$. If the paths are much longer than the circular depolarization length $\ell_{p, c}$, the handedness $\sigma$ changes sign randomly numerous times along a light path. Consequently, the average phase difference between the direct and the reversed path vanishes in the diffusion limit (path length $s \gg \ell^{\star} \approx \ell_{p, c}$ ). For the variance, however, one obtains on average over all paths (path configurations): $\left\langle(\Delta \alpha)^{2}\right\rangle=\frac{2}{3} s \ell_{\mathrm{FR}}^{\star}(2 V B)^{2}$. The new parameter $\ell_{\mathrm{FR}}^{\star}$ is the characteristic length for the influence of FR on the multiple light scattering [9]. One normally finds $2 \ell^{\star} \geq \ell_{\mathrm{FR}}^{\star} \gtrsim \ell^{\star}$. It can be shown that in the diffusion limit and for small values of $V B \ell^{\star}$, this results in a rounding of the $\mathrm{CB}$ cone and a decrease of its height according to the replacement $q_{\mathrm{b}}^{2} \rightarrow q_{\mathrm{b}}^{2}+\ell_{\mathrm{FR}}^{\star} / \ell^{\star} \cdot(2 V B)^{2}$; a behavior which has already been studied and verified experimentally elsewhere $[6,7,9-13]$.

In this letter we present experimental findings, which strongly deviate from this diffusion model. We observe that the influence of FR on CB depends on the direction of the magnetic field with respect to the direction of the incident light, and it depends on the incident and detected polarization state. We present first measurements for the case of identical incident and detected circular polarization $(++)$ as well as for the other polarization states: linear parallel $(x x)$, linear perpendicular $(x y)$ and circular orthogonal $(+-)$. By precisely evaluating these data we discover a new, unexpected effect which manifests itself in a transversal asymmetry of the $\mathrm{CB}$ cone. Its explanation will be given at the end of this letter.

The sample used was fabricated by grinding a Faraday active glass [14] and remelting the powder thus obtaining a white pellet (diameter $7 \mathrm{~mm}$, thickness $3.5 \mathrm{~mm}$ ) of a Faraday rotating bulk material containing a volume fraction $\phi \approx 5 \%$ of small air bubbles. The Verdet constant of the bulk material at the given experimental conditions (room temperature, $\lambda_{0}=458 \mathrm{~nm}$ ) was $V_{\text {bulk }}=-9.25^{\circ} / \mathrm{mm} \mathrm{T}$, i.e. we obtained in our sample a volume-averaged $\mathrm{FR}$ of $-1.15 \mathrm{rad} / \mathrm{mm}$ at $7.5 \mathrm{~T}$. We compare the shape and width of the CB cone without magnetic field and the decrease of the cone height in increasing field with Monte Carlo simulations $\left({ }^{2}\right)$. This gives a transport mean free path of $\ell^{\star}=445 \mu \mathrm{m}$ (see fig. 1). According to Mie calculations, this value corresponds to an average $(\phi=$ const) particle diameter of $20 \mu \mathrm{m}$. The Monte

\footnotetext{
$\left({ }^{1}\right)$ Due to the fact that we consider light propagation in turbid media, the bulk Verdet constant $V_{\text {bulk }}-$ corrected by the volume fraction $\phi$ of the Faraday active material - is not necessarily identical to the effective Verdet constant $V_{\text {eff }}$ which acts on the coherent beam between two scattering events [8]. However, in this as well as in former works, $V_{\text {eff }}$ was always found to be identical to $\phi V_{\text {bulk }}$ within the experimental error bars. For simplicity, in the following we will abbreviate $V_{\text {eff }}$ by $V$.

$\left({ }^{2}\right)$ References [3,9] describe the way how these Monte Carlo simulations are performed in more detail: We consider a random walk of photons with exponential path length distribution. The distribution of the scattering angles is determined by the exact scattering cross-section (without Faraday effect) of the given Mie spheres. The Faraday rotation acts on the coherent beam between two scattering events. The surface of the sample is taken into consideration using Fresnel's laws. Contrary to other works on the influence of the Faraday effect on multiple light scattering $[8,15,16]$, we neglected the influence of the Faraday effect on the scattering crosssection. This is justified by the fact that the effects described in this article are of order unity, whereas effects on the scattering cross-section are several orders of magnitude smaller. Moreover, the effects described here are real multiple scattering effects, while most of the effects described in $[8,15,16]$ can, at least qualitatively, be well understood by a magnetic-field-dependent single scattering cross-section. It is perhaps worth mentioning that, once the influence of the Faraday effect on the cross-section is known [17,18], this modified cross-section can be easily incorporated in the simulations, if desired.
} 


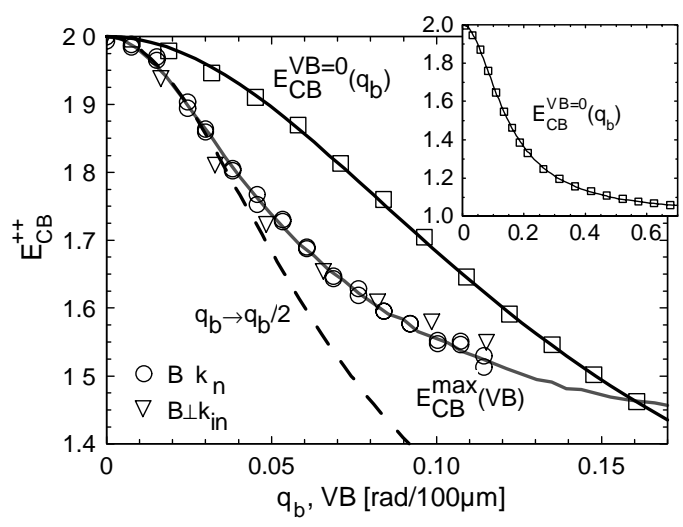

Fig. 1

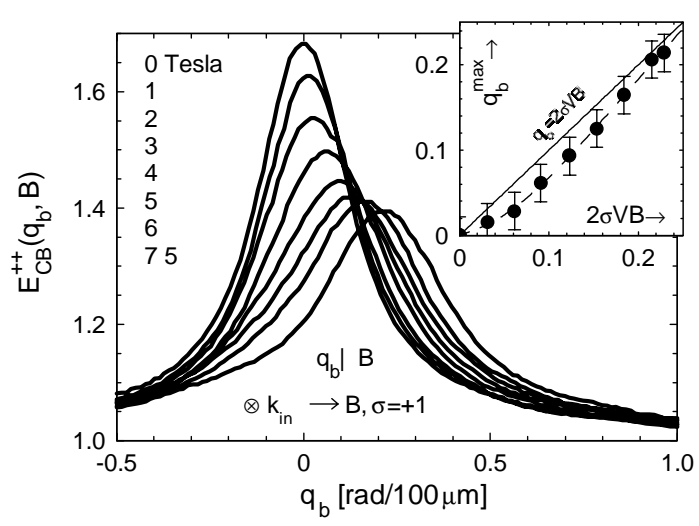

Fig. 2

Fig. 1 - Comparison of experimental data $(\square, \bigcirc, \nabla)$ with Monte Carlo simulations (solid lines) for Mie spheres. $\square$ : CB enhancement $E_{\mathrm{CB}}^{V B=0}\left(q_{\mathrm{b}}\right)$ without magnetic field as a function of $q_{\mathrm{b}} \cdot \bigcirc, \nabla$ : Decrease of the cone maximum with magnetic field $E_{\mathrm{CB}}^{\max }(V B)$. Best coincidence between experiments and simulations was found for an average sphere diameter of $20 \mu \mathrm{m}$ and $\ell^{\star}=445 \mu \mathrm{m}$. All curves were rescaled such that $E_{\mathrm{CB}}$ ranges from 2 to 1 . In the case $\vec{B} \| \vec{k}_{\text {in }}(\bigcirc)$, of course, the position of the cone maximum is unchanged; its decrease is essentially identical to that of the case $\vec{B} \perp \vec{k}_{\text {in }}(\nabla)$. The dashed line demonstrates that for small arguments (diffusion limit) the curve $E_{\mathrm{CB}}^{\max }(V B)$ can be obtained from the curve $E_{\mathrm{CB}}^{V B=0}\left(q_{\mathrm{b}}\right)$ by rescaling (see text). Inset: $\mathrm{CB}$ cone and simulation for a larger angle range. For values smaller than $0.2 \mathrm{rad} / 100 \mu \mathrm{m}$ only every second ( $>0.2$ every fourth) measured point is displayed. Sample: Faraday active bulk material containing small air bubbles; circular polarization, $\lambda_{0}=458 \mathrm{~nm}$, room temperature.

Fig. 2 - CB enhancement $\left(E_{\mathrm{CB}}\right)$ as a function of backscattering vector $q_{\mathrm{b}}$ (more precisely, the part of $\vec{q}_{\mathrm{b}}$ which is parallel to $\vec{B}$ ) for different magnetic-field values between 0 and 7.5 T. Same incident and detected circular polarization state " ++ " and $\vec{B} \perp \vec{k}_{\mathrm{in}}$. With increasing field the cone height decreases to some extent and the maximum is shifted off the exact backscattering direction. Inset: shift of the cone maximum $q_{\mathrm{b}}^{\max }$ as a function of $2 V B$. With increasing FR both values become equal, as predicted. The deviation at smaller values originates from the superimposed decrease of the cone.

Carlo simulations have been carried out using these parameters (Mie scattering, ratio of index of refraction $m=1 / 1.7$, etc.) by additionally taking into consideration the finite sample thickness, a limited experimental resolution of about $5 \cdot 10^{-5} \mathrm{rad}$ and internal reflections at the surfaces of the sample $\left({ }^{3}\right)$. From the differential cross-section of the Mie scatterers one can calculate the lengths $\ell^{\star}, \ell_{p, c}$ and $\ell_{\mathrm{FR}}^{\star}$ according to the following equations [9]:

$$
\ell^{\star}=\frac{\ell}{1-\langle\cos \theta\rangle}, \quad \ell_{p, c}=\frac{-\ell}{\ln \left(\frac{\left\langle I(\theta)^{+}-I(\theta)^{-}\right\rangle}{\left\langle I(\theta)^{+}+I(\theta)^{-}\right\rangle}\right)}, \quad \ell_{\mathrm{FR}}^{\star}=\frac{\ell}{1-\frac{\left\langle\left(I(\theta)^{+}-I(\theta)^{-}\right) \cos \theta\right\rangle}{\left\langle I(\theta)^{+}+I(\theta)^{-}\right\rangle}},
$$

where $\ell$ is the scattering mean free path and $I(\theta)^{+}, I(\theta)^{-}$are the light intensities scattered (by a single Mie sphere) into the same or opposite circular polarization state, respectively, at a certain scattering angle $\theta$. The averages are taken over all scattering directions. In our case we obtain $\ell_{p, c}=1.962 \ell^{\star}, \ell_{\mathrm{FR}}^{\star}=0.955 \ell^{\star}$ and $\ell^{\star}=3.522 \ell$.

\footnotetext{
$\left({ }^{3}\right)$ By varying the strength of the internal reflections, we found that the measured curves could be fitted best for an index mismatch at the surface of only $m_{\mathrm{s}}=1 / 1.3$. This might be explained by the roughness of the surface.
} 


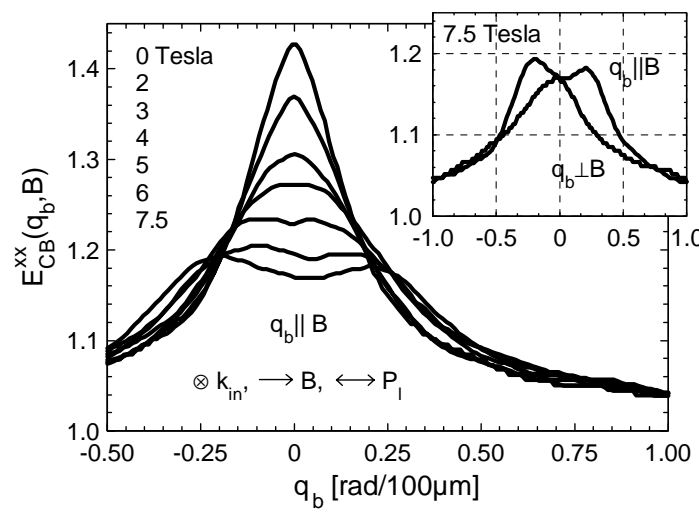

Fig. 3

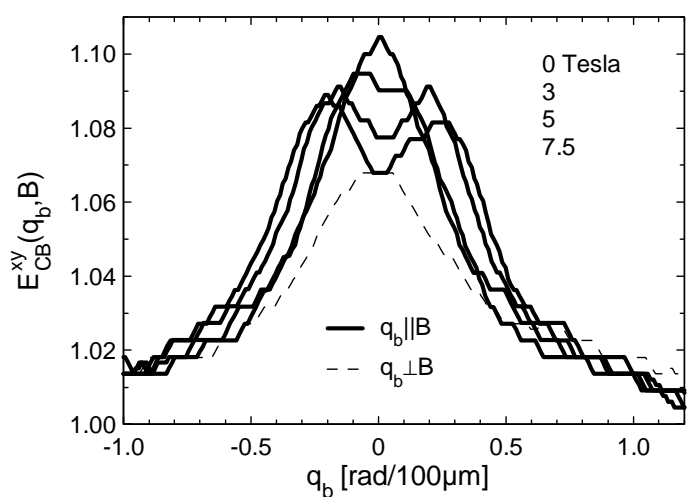

Fig. 4

Fig. 3 - Linear polarization, parallel polarizer and analyzer, $\vec{B} \perp \vec{k}_{\text {in }}$. CB enhancement as a function of the component of $\vec{q}_{\mathrm{b}}$ that is parallel to $\vec{B}$ for different field values. The inset shows the curve at 7.5 T once again as well as the $\mathrm{CB}$ enhancement in the direction perpendicular to $\vec{B}\left(\vec{q}_{\mathrm{b}} \perp \vec{B}\right)$.

Fig. 4 - Same situation as in fig. 3 but for crossed linear polarizer and analyzer $(x y)$.

In backscattering geometry about $50 \%$ of the light paths are shorter than $10 \ell^{\star}$ and thus only poorly described by the diffusion approximation. In fact, for the part of the light where the circular polarization is not destroyed $\left(s \lesssim \ell_{p, c}\right)$, the phase difference between the direct and the reversed path is $\Delta \alpha=2 \sigma V \vec{B} \cdot \vec{R}$. Consequently, if the magnetic field is parallel to the incident light, $\vec{k}_{\text {in }} \| \vec{B}\left(\Rightarrow \vec{R} \perp \vec{B}\right.$ and $\left.\vec{q}_{\mathrm{b}} \perp \vec{B}\right)$, there will be no phase difference in the exact backscattering direction, i.e. the coherence between a pair of paths for this part of the light will not be influenced by FR.

In the case $\vec{B} \perp \vec{k}_{\text {in }}$, the phase shift $\Delta \alpha$ can be compensated by a corresponding phase shift $\vec{q}_{\mathrm{b}}^{\prime} \cdot \vec{R} \stackrel{!}{=}-2 \sigma V \vec{B} \cdot \vec{R}$ at a certain angle $\vec{\theta}_{\mathrm{b}}^{\prime}=\vec{q}_{\mathrm{b}}^{\prime} \lambda_{0} / 2 \pi$ off exact backscattering, in a direction parallel to the magnetic field $\left(\vec{q}_{\mathrm{b}}|| \vec{B}\right)$. Consequently, for the non-depolarized part of the light, the $\mathrm{CB}$ cone is not destroyed but shifted off the exact backscattering direction according to the replacement $\vec{q}_{\mathrm{b}} \rightarrow \vec{q}_{\mathrm{b}}+2 \sigma V \vec{B}$. Figure 2 shows the first measurement which clearly demonstrates this behavior.

In the case of linearly polarized incident and detected light " $x x$ "' and $\vec{k}_{\text {in }} \perp \vec{B}$, two maxima appear at $\vec{q}_{\mathrm{b}}= \pm 2 V \vec{B}$ into and opposite to the direction of the magnetic field (see figs. 3, 5, 6). This behavior can be explained by decomposing the linear polarization into the two circular polarized states $\sigma= \pm 1\left({ }^{4}\right)$. There is also a transversal asymmetry visible (inset of fig. 3 and fig. 6) which will be discussed further below. In the case of crossed linear analyzer and polarizer " $x y$ " a twin peak appears as well (see fig. 4). The argumentation for this effect is the same as in the case " $x x$ ".

If the magnetic field is parallel to $\vec{k}_{\text {in }}$, in the case of linear polarization (no figure), the maximum and minimum values of the $\mathrm{CB}$ enhancement are no longer found for parallel or orthogonal linear polarizer and analyzer, respectively. This effect can be explained by the penetration of the light through a layer at the surface, where the light transport is not yet diffuse. The propagation of the light through this surface layer leads to a common Faraday

$\left({ }^{4}\right)$ This argumentation is only correct, as, due to the tortuosities of the multiple scattered light paths, both polarization states become decorrelated. Without this decorrelation the large central peak would not be destroyed and only two smaller secondary maxima would appear at $\pm 2 \mathrm{VB}$. 


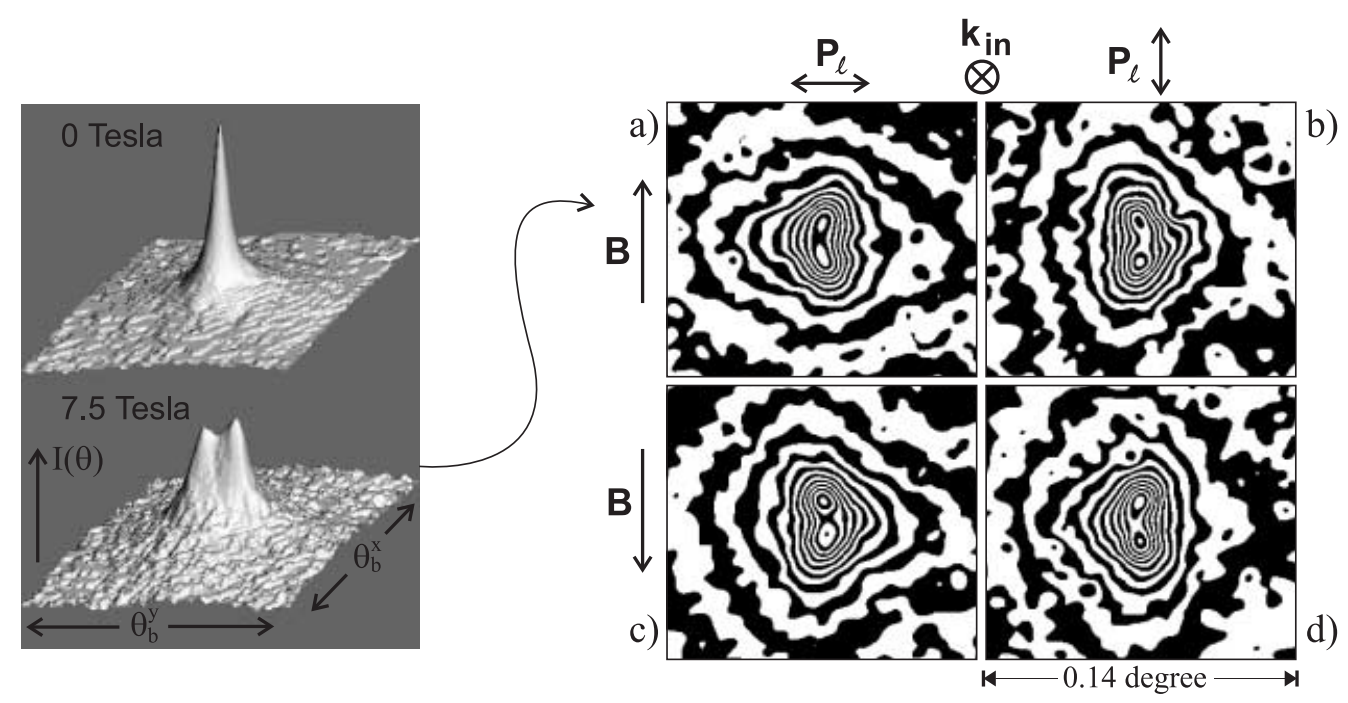

Fig. 5

Fig. 6

Fig. $5-2 \mathrm{D}$ intesity profile plot of fig. 3 . The formation of a twin peak at $7.5 \mathrm{~T}$ is clearly visible.

Fig. 6 - a) Contour plot of fig. 5. A transversal asymmetry is clearly visible. b)-d) Equivalent measurements for different combinations of the orientations of $\vec{B}$ and polarization $\vec{P}_{1}$.

rotation of the incident and outgoing wave which can be compensated by a rotation of the analyzer (for a more detailed description the reader is referred to [9]). We found a thickness of this layer of $625 \mu \mathrm{m}=1.4 \ell^{\star}$ compared to $0.93 \ell^{\star}$ in the simulations. This larger value can be explained by an air bubble free glass layer on the surface originating from the fabrication procedure. After compensation of this rotation we measured a Faraday-induced decrease of the cone maximum from 1.51 to 1.26 and an increase of the minimum value with field from 1.095 to 1.135 .

In the case of orthogonal circular incident and detected polarization states "+-" (no figure), for both $\vec{B} \| \vec{k}_{\text {in }}$ and $\vec{B} \perp \vec{k}_{\text {in }}$, we measured a CB enhancement of about 1.1 which was unchanged under magnetic field. This behavior was predicted and explained in more detail elsewhere $[7,9]$.

Figure 6 shows a contour plot of figs. 3 and 5 as well as three further measurements for different combinations of the magnetic-field direction and linear polarization $\vec{P}_{1}$ of the incident light. Note that neither $\vec{B}, \vec{P}_{1}, \vec{k}_{\text {in }}$ nor the multiple light scattering process itself have an intrinsic asymmetry. Nevertheless, a transversal ("bean-like") asymmetry in the backscattered photon flux is clearly visible which changes its orientation in dependence of the directions of $\vec{B}$ and $\vec{P}_{1}$. This asymmetry could also be confirmed by Monte Carlo simulations (see fig. 8). We found that it can be explained by the differential cross-section of Mie spheres whose index of refraction is smaller than that of the bulk material, i.e. $m<1$. The scattering of such spheres is - to some extent- comparable to a birefringent material (see inset of fig. 7$)$ : Incident light with a linear polarization $( \pm 1,1)$, i.e. a polarization which includes an angle of $45^{\circ}$ with the scattering plane, is elliptically polarized after scattering $\left({ }^{5}\right)$. In order to

$\left({ }^{5}\right)$ We have chosen a right-handed coordinate system with the first component perpendicular to the scattering plane, the second component in this plane and the third component in the direction of propagation. 


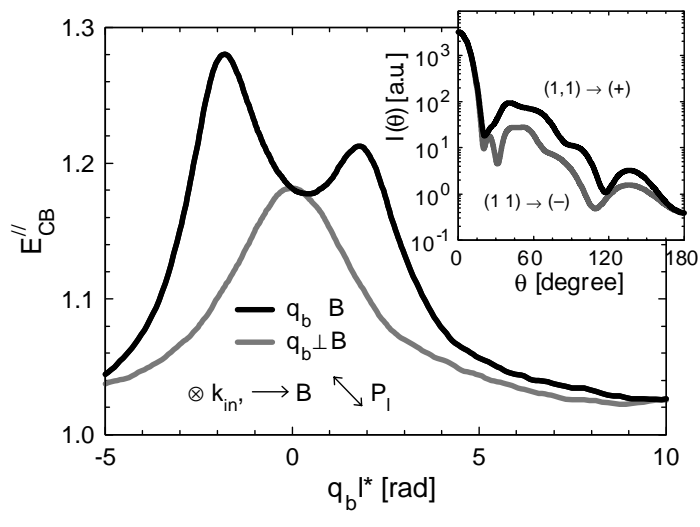

Fig. 7

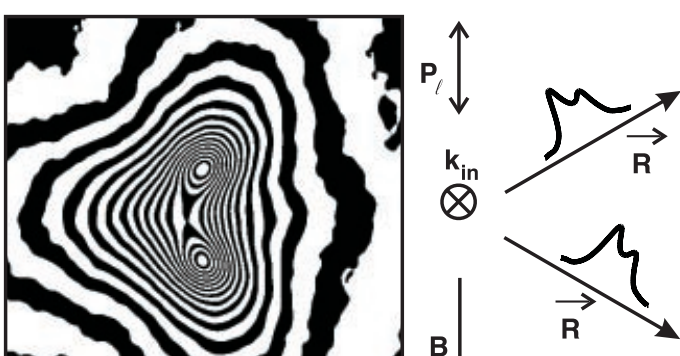

Fig. 8

Fig. 7 - Monte Carlo simulation of $E_{\mathrm{CB}}$ for Mie scattering with linear incident and detected polarization $\vec{P}_{1}=(-1,1)$. Particle diameter $=1 \mu \mathrm{m}, m=1 / 1.7, \lambda_{0}=458 \mathrm{~nm}, V B \ell^{\star}=-1$, absorption length $\ell_{\mathrm{a}}=100 \ell^{\star}$. In the simulations, FR is only introduced between the scattering events, the cross-section for the Mie scattering (inset) was considered to be field independent. In this part of the simulations, surface effects have not been taken into consideration.

Fig. 8 - Monte Carlo simulation of the transversal asymmetry of the cone. Essentially the same parameters as in fig. 7 but for $\vec{P}_{1} \| \vec{B}$ and for particles with a diameter of $20 \mu \mathrm{m}$.

understand the asymmetry in fig. 6, let us first consider another situation, denoted by "\|", which is essentially the same situation as in fig. 3, i.e. $\vec{B} \perp \vec{k}_{\text {in }}$ and $\vec{q}_{\mathrm{b}} \| \vec{B}$, but for an incident and detected polarization $\vec{P}_{1}=(-1,1)$. Moreover, let us consider light paths which propagate more or less in the plane given by $\vec{k}_{\text {in }}$ and $\vec{q}_{\mathrm{b}} \| \vec{B}$, such that the scattering plane of the first and last scattering event include an angle of $45^{\circ}$ with $\vec{P}_{1}$. In this case, as mentioned above, the light becomes elliptically polarized after the first scattering event. With magnetic field, in analogy to figs. 2 and 3, the linear part of the elliptically polarized light is split into a twin peak and the circular part is shifted in the direction of $\sigma V \vec{B}$, respectively. The result is an asymmetric twin peak as can be seen in fig. 7. Considering the case "|l", it is now possible to understand the asymmetry of figs. 6 and 8: Light paths that essentially propagate in a plane that includes an angle of $\pm 45^{\circ}$ with respect to $\vec{P}_{1}$ and $\vec{k}_{\text {in }}$ correspond to some extent to the case "I", as indicated in the sketch on the right side of fig. 8. Surprisingly, both the plane that includes an angle of $45^{\circ}$ and the plane that includes an angle of $-45^{\circ}$ have a higher left maximum originating from the fact that from one case to the other both products $\vec{B} \cdot \vec{R}$ and $\vec{P}_{1} \cdot \vec{R}$ change sign (the latter corresponds to a replacement $(-1,1) \rightarrow(1,1)$ in fig. 7 ). This results in a transversal asymmetry of the cone. This simple model is consistent with the measurements of fig. 6a)-d) where the direction of the asymmetry depends on the incident polarization, $(1,0)$ or $(0,1)$, and it is consistent with the fact that there is no asymmetry in the linear crossed case " $x y$ " (no figure).

In the simulations we also found a transversal asymmetry (however about 2 orders of magnitude smaller) in the case of Rayleigh-Gans-Debye scattering when we additionally included a magnetic-field-induced birefringence (the so-called Voigt effect which is a concomitant of FR). This magneto-optical birefringence has a similar effect as the "local birefringence" of Mie scattering, for example a cone comparable to that of fig. 7 is obtained as well. In the case of Rayleigh-Gans-Debye scattering, however, the strength of the asymmetry was different for 
the cases $\vec{P}_{1} \| \vec{B}$ and $\vec{P}_{1} \perp \vec{B}$ (i.e. cases corresponding to fig. 6a) and b), which is probably due to the fact that, in the case of Rayleigh-Gans-Debye scattering, the CB cone (without magnetic field) is not rotational invariant.

Recently, a very small transversal asymmetry of the photon flux was observed, in an "optical Hall effect" [15]. This effect was explained in a completely different way, by a magneticfield-induced change of the differential cross-section of the scatterers. (In our case, the magnetic field is assumed to only affect the coherent wave between two scattering events.) These experiments had been carried out with unpolarized light. In our case, according to the superposition of fig. 6a) and b), there would be no net effect for unpolarized light, at least for Mie scattering. In the case of Rayleigh-Gans-Debye scattering, where the cases "6a)" and "6b)" are not totally symmetric as explained above, a small transversal asymmetry could possibly survive for unpolarized light. In future work, a possible relation between the optical Hall effect and the transversal asymmetry described here, should be studied in more detail. In particular, it remains unclear why the calculations in [8], using a Green's function formalism to describe magnetic-field effects on coherent backscattering, completely disagree with our experimental findings.

\section{REFERENCES}

[1] van Albada M. P. and LagendiJk A., Phys. Rev. Lett., 55 (1985) 2692.

[2] Wolf P.-E. and Maret G., Phys. Rev. Lett., 55 (1985) 2696.

[3] Lenke R. and Maret G., Scattering in Polymeric and Colloidal Systems, edited by W. Brown and K. Mortensen (Gordon and Breach Scientific) 2000, p. 1.

[4] Lagendijk A. and van Tiggelen B. A., Phys. Rep., 270 (1996) 143.

[5] Van Rossum M. C. W. and Nieuwenhuizen Th. M., Rev. Mod. Phys., 71 (1999) 313.

[6] Golubentsev A. A., Sov. Phys. JeTP, 59 (1984) 26.

[7] Mackintosh F. C. and John S., Phys. Rev. B, 37 (1988) 1884.

[8] Lacoste D. and van Tiggelen B. A., Phys. Rev. E, 61 (2000) 4556.

[9] Lenke R. and Maret G., Eur. Phys. J. B, 17 (2000) 171.

[10] Erbacher F. A., Lenke R. and Maret G., Europhys. Lett., 21 (1993) 551.

[11] Lenke R. and Maret G., Phys. Scr. B, T49 (1993) 605.

[12] Lenke R. and Maret G., OSA Proceedings on Advances in Optical Imaging and Photon Migration, Vol. 21, edited by R. R Alfano (Optical Society of America) 1994, p. 16.

[13] Martinez A. S. and Maynard R., Phys. Rev. B, 50 (1994) 3714.

[14] FR-5 Terbium Borosilicate Glass (paramagnetic), manufacturer Hoya.

[15] Rikken G. L. J. A. and van Tiggelen B. A., Nature, 381 (1996) 54.

[16] Sparenberg A., Rikken G. L. J. A. and van Tiggelen B. A., Phys. Rev. Lett., 79 (1997) 757.

[17] Rikken G. L. J. A. and van Tiggelen B. A., Phys. Rev. Lett., 78 (1997) 847.

[18] Lacoste D. and van Tiggelen B. A., J. Opt. Soc. Am. A, 15 (1998) 1636. 